\title{
Ausschreibung des Gustav-Bucky-Preises 2017
}

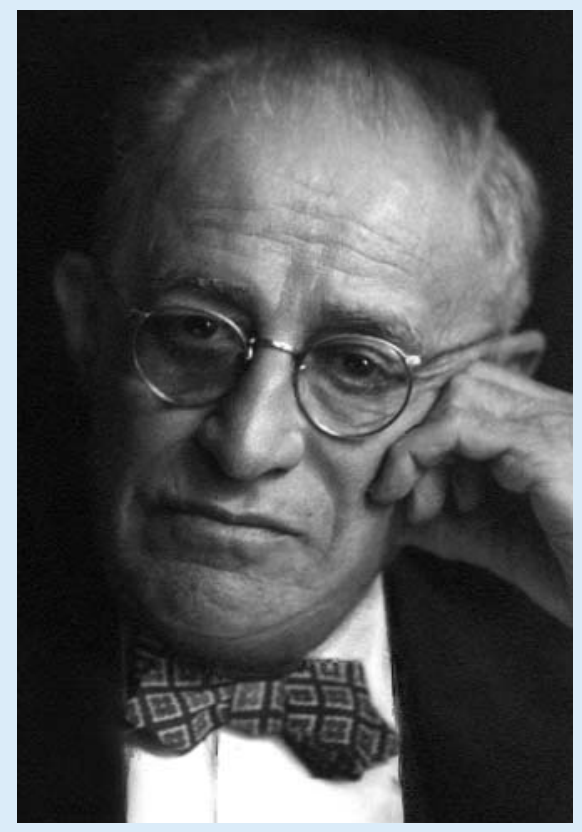

Gustav Bucky (Bildquelle: Archiv Deutsches Röntgen-Museum)

Die Berliner Röntgengesellschaft - Röntgenvereinigung zu Berlin und Brandenburg e. V. schreibt für 2017 den Gustav-BuckyPreis aus. Der Gustav-Bucky-Preis wird mit dem Ziel vergeben, den radiologischen wissenschaftlichen Nachwuchs zu fördern und ist mit 500 Euro dotiert.

\section{Bewerbung}

Ärzte und Naturwissenschaftler können sich mit einer wissenschaftlichen, klinischen Originalarbeit zum Thema Radiologie bewerben. Die Arbeit muss im Jahr 2016 bis 2017 publiziert oder zur Publikation ange- nommen sein. Nachweise für zur Publikation angenommene Arbeiten sind zu erbringen. Promovenden, die zwischen dem 01.01.2016 und dem 31.06.2017 ihre Promotion abgeschlossen haben, können sich mit ihrer Dissertation bewerben. Der Bewerber sollte Erstautor der eingereichten Arbeit und nicht älter als 35 Jahre sein. Bei geteilter Erstautorenschaft muss die Bewerbung von beiden Erstautoren erfolgen.

Bewerbungsfrist: 30. September 2017

\section{Unterlagen}

Senden Sie bitte die folgenden Unterlagen in 2-facher Ausfertigung an:

Den 1. Vorsitzenden der Berliner Röntgengesellschaft - Röntgenvereinigung zu Berlin und Brandenburg e. V.:

PD Dr. med. Timm Denecke, Klinik für Radiologie, Campus Virchow-Klinikum, Charité - Universitätsmedizin Berlin, Augustenburger Platz 1, 13353 Berlin

- Anschreiben

- Lebenslauf (ggf. Publikationsverzeichnis)

- Erklärung „Hiermit bestätige ich, dass die Koautoren mit der Bewerbung einverstanden sind sowie die wissenschaftliche Arbeit nicht parallel zu einer anderen wissenschaftlichen Preisverleihung eingereicht oder bereits prämiert wurde."

- Wissenschaftliche Originalarbeit

\section{Verleihung}

Die Preisverleihung findet im Rahmen einer wissenschaftlichen Sitzung der Gesellschaft (voraussichtlich im November) im Hörsaal der Kaiserin-Friedrich-Stiftung am RobertKoch-Platz 7 in 10117 Berlin statt.

\section{Zur Person}

Gustav Bucky wurde am 3. September 1880 in Leipzig geboren. Er publizierte über 100 wissenschaftliche Arbeiten und besaß 94 deutsche und amerikanische Patente auf dem Gebiet der Röntgentechnik. Bekannt wurde sein Name vor allem durch die Erfindung der nach im benannten Bucky-Blende, einem wichtigen Beitrag zur Reduktion der Streustrahlung und Qualitätsverbesserung in der Röntgendiagnostik. 1933 emigrierte Bucky von Berlin nach New York. Dort leitete er die physiotherapeutische Abteilung des Sea View Hospitals, unterhielt ein eigenes Röntgenlaboratorium und wurde Hochschullehrer am New York College of Medicine sowie am Albert Einstein College of Medicine. Gustav Bucky starb 1963 in New York. Die Röntgenvereinigung zu Berlin und Brandenburg ehrt mit diesem Preis Gustav Bucky sowie seine radiologischen Kollegen, die zwischen 1933 und 1945 aus ihrer Tätigkeit verbannt oder zur Emigration gezwungen wurden.

Der Vorstand der Berliner Röntgengesellschaft - Röntgenvereinigung zu Berlin und Brandenburg e. V.

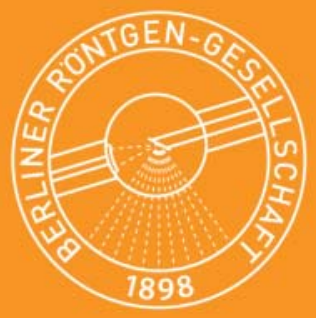

